\title{
APRENDIZAJE POR AUTORREGULACIÓN EN UN CENTRO RURAL AGRUPADO
}

Self-regulated learning in a grouped rural center

Auto-regulação da aprendizagem em uma escola rural agrupados

\author{
Hugo Medina Melcón (1)
}

Laura Álvarez Cañas (2)

(1) Centro Rural Agrupado Vega del Esla, Toral de los Guzmanes, León, España. Teléfono: +34 620540172. Correo electrónico: hugo.opositta@gmail.com

(2) Centro Rural Agrupado Ruta de la Plata, León, España. Teléfono: +34 676676655. Correo electrónico: guasariiii@hotmail.com

\section{Resumen}

Esta comunicación presenta una solución metodológica de trabajo en las aulas incompletas de los centros rurales agrupados. Lejos de buscar la homogenización de los aprendizajes entre los distintos cursos presentes en dichas aulas, a continuación, se plantea el trabajo individual y cooperativo para buscar el desarrollo de la responsabilidad personal y autonomía a través del Aprendizaje Autorregulado. El área de Lengua Castellana y Literatura de la etapa de Educación Primaria del Sistema Educativo Español, se presenta como ideal para comenzar a establecer un cambio de mentalidad y forma de trabajar ya que por sus características es un área privilegiada para fomentar la participación del alumnado en el grupo-clase de modo individual y cooperativo. Los resultados conseguidos han ido más allá de lo esperado, generándose dinámicas intragrupales específicas que hacen que el trabajo de los alumnos sea más eficaz.

Palabras clave: Aprendizaje Autorregulado; Autonomía; Evaluación formativa; Aulas Incompletas

\begin{abstract}
The present paper presents a methodological solution of work in the incomplete classrooms in a Rural Grouped Center. Far from looking for the homogenization of learning between the different courses present in these classrooms, then, the individual Aprendizaje por autorregulación en un centro rural agrupado
\end{abstract}


and cooperative work will be proposed to seek the development of personal responsibility and autonomy through self-regulated learning. The Castilian Language and Literature area of the Primary Education stage of the Spanish Educational System is presented as an ideal to begin to establish a change of mentality and way of working because of its characteristics is a privileged area to encourage student participation in the group-class individually and cooperatively. The results achieved have gone beyond expectations, generating specific intra-group dynamics that make the work of students more effective.

Keywords: Self-regulated learning; Autonomy; Formative evaluation; Incomplete Classes

\section{Resumo}

Este artigo apresenta uma solução de trabalho metodológico nas salas de aula incompletas agrupados centros rurais. Longe de buscar a padronização de aprendizagem entre diferentes cursos presentes nestas salas de aula, em seguida, trabalho individual e cooperativa terá como objetivo buscar o desenvolvimento de responsabilidade pessoal e de autonomia através da aprendizagem auto-regulada. A área de Língua Espanhola e Literatura na fase de Ensino Primário Sistema espanhol, é apresentado como um ideal para começar a estabelecer uma mudança de mentalidade e forma de trabalhar e que por sua natureza é uma área privilegiada para incentivar a participação dos alunos em grupo de classe individual e cooperativamente. Os resultados alcançados foram além das expectativas, gerando dinâmicas específicas dentro do grupo que fazem trabalhar a os estudantes mais eficazmente.

Palavras-chave: Auto-regulação da aprendizagem; autonomia; avaliação formativa; Salas de aula Incompletas

\section{Introducción}

Tradicionalmente en las aulas incompletas de los Centros Rurales Agrupados (LópezPastor, 1999) se ha tendido a homogeneizar las enseñanzas para intentar que los alumnos realizaran un mayor aprovechamiento del tiempo de clase. Esto provocaba que los pequeños parecieran adelantados al nivel de contenidos propio de su curso y edad, y que los mayores de la clase profundizaran poco en esos mismos contenidos, lo que 
desembocaba en una falta de individualización del proceso de enseñanza aprendizaje en contextos que eran muy propicios para que existiese.

Esta propuesta está basada en los procesos de aprendizaje por Autorregulación que se revelan como una metodología de transición entre los modelos tradicionales de enseñanza-aprendizaje y aquellas propuestas basadas en el Aprendizaje Cooperativo o Aprendizaje Basado en Proyectos (Casado, Pérez-Pueyo \& Casado, 2017) y para ser llevada a cabo en el área de Lengua Castellana y Literatura de la Etapa de Educación Primaria.

\section{Contextualización}

La experiencia se ha llevado a cabo en un aula incompleta de primaría del CRA Vega del Esla, formada por ocho alumnos que cursan $2^{\circ}, 3^{\circ}, 4^{\circ}$ y $5^{\circ}$, de los cuales cinco son de $3^{\circ}$, presentando diversas circunstancias personales y familiares que condicionan su rendimiento académico y su ritmo de aprendizaje. Así mismo, se cuenta con un alumno de $4^{\circ}$ diagnosticado dentro de la categoría de Capacidad Intelectual Límite según la normativa de Castilla y León (2015) y se completa con dos alumnas de $2^{\circ}$ y $5^{\circ}$ respectivamente que presentan buen ritmo y estilo de aprendizaje.

\section{Diseño y desarrollo}

Las principales ventajas del Aprendizaje Autorregulado, como metodología de transición entre los modelos tradicionales y las metodologías activas (Casado, PérezPueyo \& Casado, 2017; Zimmerman, 2002), se encuentran en que sin abandonar el uso de los libros de texto, estos se utilizan como recurso de consulta y base de aprendizaje. Además, éste fomenta la autonomía del alumno respetando el ritmo y estilo individual de aprendizaje de cada uno.

La puesta en práctica de dicha metodología en el área de Lengua Castellana y Literatura requiere de la construcción de materiales graduados para cada curso, los cuales van a permitir al alumnado planificar individualmente su trabajo y desarrollarlo de forma autónoma con el apoyo del profesor.

En el plan o ficha de autorregulación que deben utilizar cada alumno debe figurar el trabajo que deben realizar (parte superior en la que establece el profesor las actividades concretas a realizar), disponiendo de un espacio para distribuirlo (parte intermedia de la ficha en la que aparece el calendario) y otro para valorar cada día su 
grado de cumplimiento de las condiciones de la autorregulación (parte inferior). En la Figura 1 se puede ver un ejemplo para los alumnos de $3^{\circ}$.

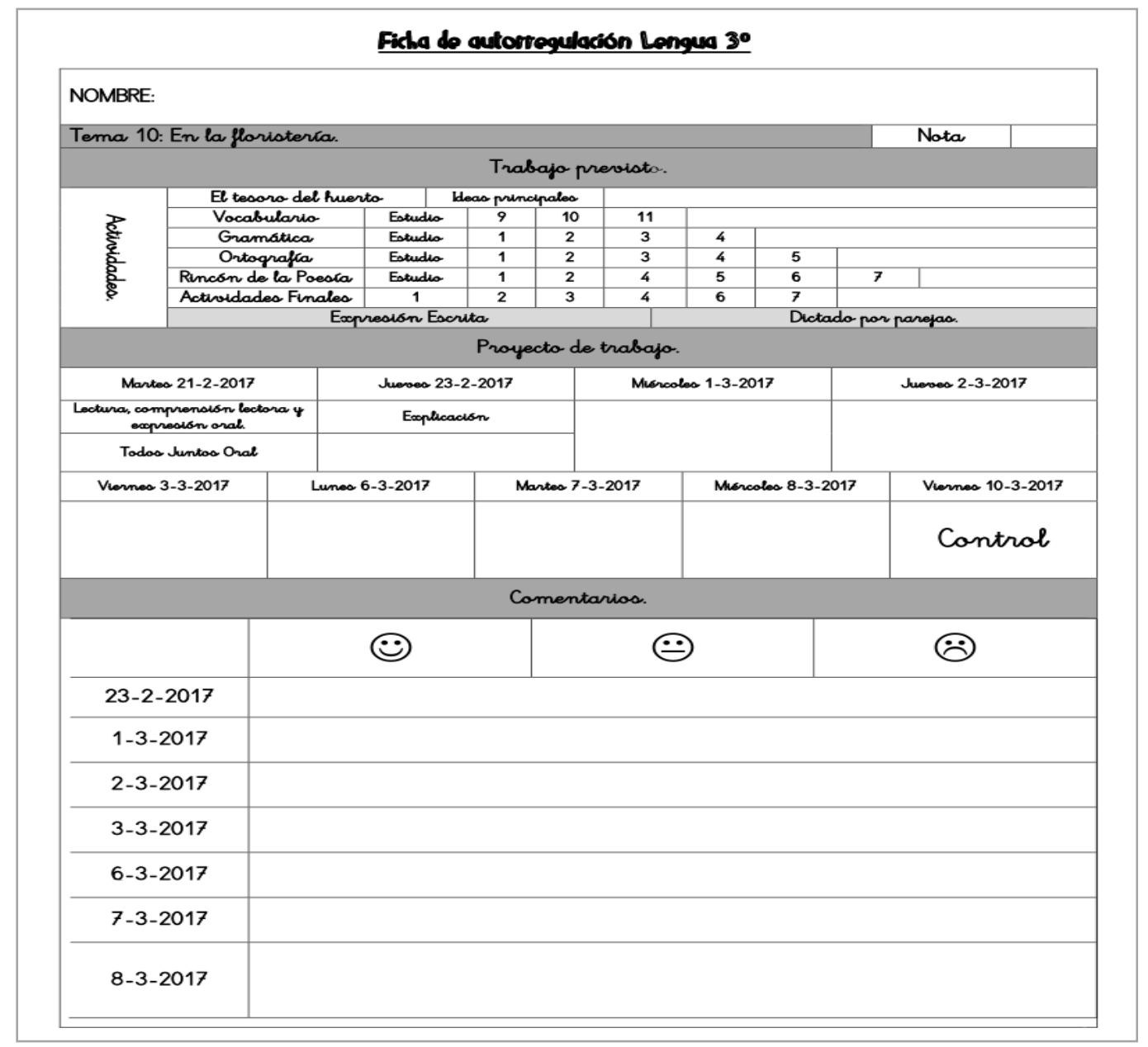

Figura 1.

Ficha de autorregulación (modificado de Casado, 2014a)

Sin embargo, además de las actividades que se realizan del libro y que se abordan de manera individual generalmente, el docente debe diseñar otras cuyas características tengan un carácter verdaderamente competencial y que requieran de la coordinación con otros compañeros como las fichas de expresión escrita (figura 2) o de dictado por parejas (Casado, 2014b; Casado \& Pérez-Pueyo, 2017); actividades que deben ubicar también en alguno de los días. En este caso deben ponerse de acuerdo con otro compañero para ubicarlas en sus respectivas fichas de autorregulación al tratarse de una actividad cooperativa.

Después de una explicación general de la teoría realizada el primer día, el alumno debe desarrollar el trabajo que ha planificado el resto de las sesiones en las 
cuales todos los días tendrá un tiempo de corrección y explicación de conceptos de manera individual.

De este modo cada alumno comparte espacio, tiempo y método de trabajo en el aula, pero con actividades adecuadas a sus características psicoevolutivas compartiendo algunas que deben realizar de modo cooperativo como el dictado por parejas

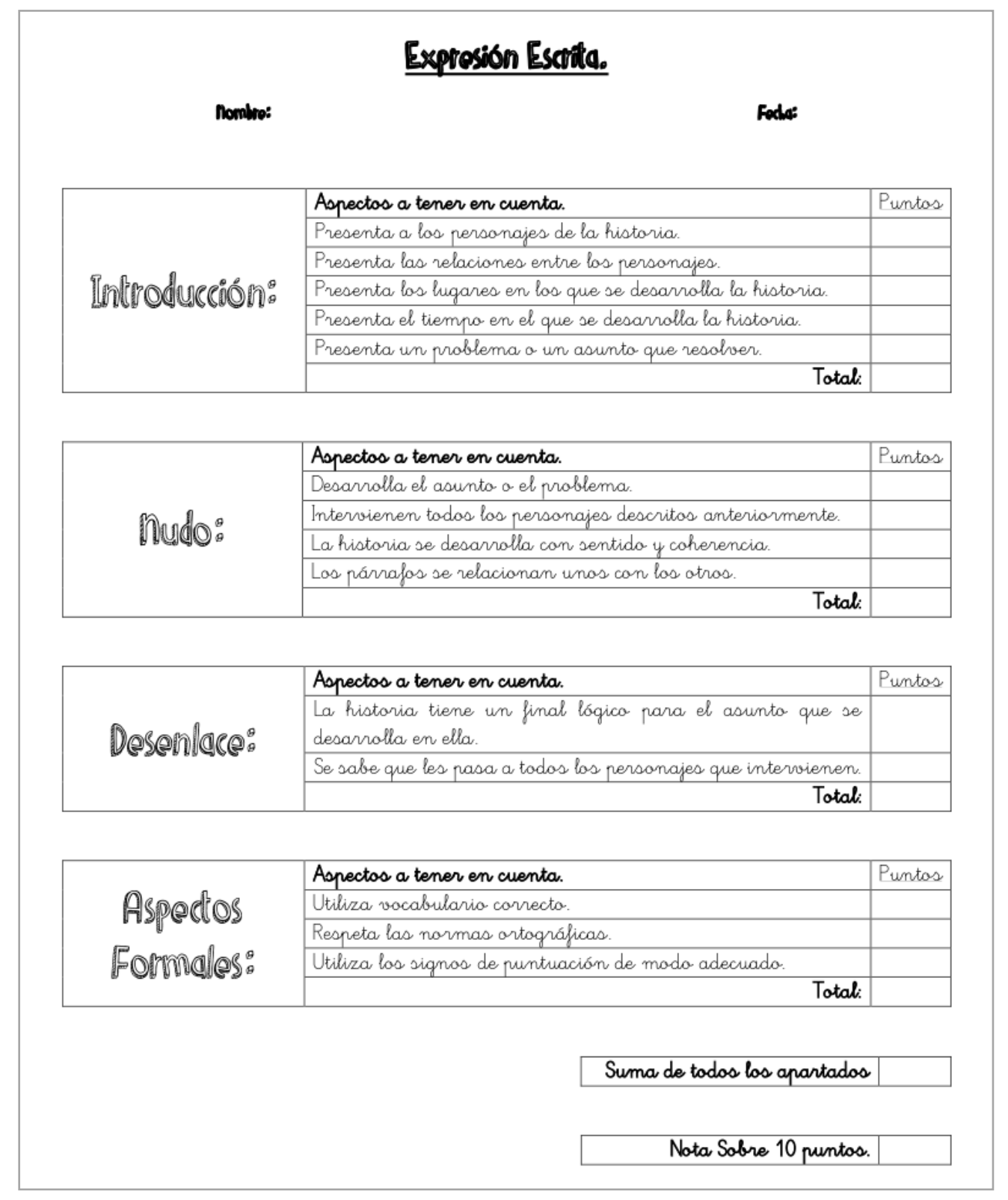

Figura 2.

Ficha Expresión Escrita (elaboración propia)

\section{Evaluación}

Desde una visión formativa, en primer lugar y en cuanto al aprendizaje y mejora de la ortografía, en cada actividad en la que se trabaje la expresión escrita siempre hay una Aprendizaje por autorregulación en un centro rural agrupado 
corrección por parte del profesor que señalará en cada línea del escrito cuáles son las faltas de ortografía que se han cometido en ella. Esta corrección no es finalista y ya que el alumno tendrá hasta tres oportunidades para modificar dicho escrito y mejorarlo, siendo en esa tercera ocasión cuando se establece la evaluación final del trabajo y calificación si procede (Casado \& Pérez-Pueyo, 2017).

Así mismo, a la hora de trabajar la expresión escrita espontanea, los alumnos disponen de una ficha (figura 2) que establece cuáles son las partes de cualquier historia (introducción, nudo y desenlace) que deberán tener en cuenta para componer su escrito y los aspectos formales ortográficos, de vocabulario y puntuación que también se han de tener en cuenta. Al igual que con la actividad anterior, el alumno tiene tres oportunidades para mejorar su trabajo siendo la función del maestro indicarle a que aspectos de la ficha no se ha ceñido y harán mejorar el resultado final.

\section{Conclusiones}

Como conclusión, la primera consideración necesaria es que el trabajo que se ha expuesto requiere paciencia y tiempo, aunque menos del que parece, ya que los alumnos deben adquirir e interiorizar los procedimientos de trabajo que implica la autorregulación y que requiere que su autonomía vaya creciendo.

Por otro lado, también es oportuno apuntar que los procesos de autorregulación están "vivos" y evolucionan conforme evoluciona la capacidad de trabajo de cada alumno y la clase como grupo.

Finalmente, la experiencia ha permitido comprobar que, aunque en clase están presentes alumnos de distintos cursos que deben trabajar de modo autónomo, todos terminan entendiendo esta metodología de trabajo de un modo grupal y se desarrollan dinámicas colectivas de aprendizaje recíproco llegando un punto en que los compañeros buscan no solo la ayuda o explicación del maestro, sino también la de compañeros que ya han realizado la actividad a la que ellos se enfrentan en ese momento; además de la ayuda de los mayores a los más pequeños.

\section{Referencias}

Casado, O. (2014a). Plan de autorregulación. Recuperado de https://drive.google.com/file/d/0B7xEdPlO4dj8U1VuS1R2RIM4NEk/view 
Casado, O. (2014b). Dictado en parejas. Recuparado de https://drive.google.com/file/d/0B7xEdPlO4dj8ZVRuMGpHYkhOT3c/view

Casado, O., \& Pérez-Pueyo, A. (2017). El aprendizaje de la ortografía a través de evaluación formativa (pp. 194-206). En V. M. López-Pastor y A. Pérez-Pueyo (coords.) Evaluación formativa y compartida en Educación: experiencias de éxito en todas las etapas educativas. León: Universidad de León.

Casado, O., Pérez-Pueyo, A. \& Casado, P. (2017). La autorregulación en educación primaria. Una propuesta para favorecer la autonomía del alumnado (pp. 208-237). En V. M. López-Pastor y A. Pérez-Pueyo (coords.) Evaluación formativa y compartida en Educación: experiencias de éxito en todas las etapas educativas. León: Universidad de León.

Instrucción de 9 de julio de 2015 de la Dirección General de Innovación Educativa y de Formación del Profesorado, por la que establecen los procedimientos de recogida y tratamiento de datos relativos a los alumnos con necesidad específica de apoyo educativo escolarizado en Castilla y León. Recuperado de http://madrastra.es/wpcontent/uploads/2015/03/Instrucci\%C3\%B3n-alumnado-necesidad-apoyoeducativo-09072015-2.pdf

López-Pastor, V. M. (Coord.) (1999). La Educación Física en la Escuela Rural. Buenos Aires: Miño y Dávila.

López-Pastor, V. \& Pérez-Pueyo, A. (2017). Evaluación formativa y compartida en evaluación formativa y compartida en educación: Experiencias de Éxito en todas las etapas educativas. Universidad de León: Servicio de Publicaciones.

Orden ECD/65/2015, de 21 de enero, por la que se describen las relaciones entre las competencias, los contenidos y los criterios de evaluación de la educación primaria, la educación secundaria obligatoria y el bachillerato (B.O.E., 29 de enero).

Real Decreto 126/2014, de 28 de febrero, por el que se establece el currículo básico de la Educación Primaria (B.O.E. 1 de marzo).

Zimmerman, B. J. (2002). Becoming a self-regulated learner: an overview. Theory into practice, $41(2), 64-70$. 